\title{
International Students' Perceptions of the Value of U.S. Higher Education
}

\author{
Ewa Urban \\ Western Michigan University (USA) \\ Louann Bierlein Palmer \\ Western Michigan University (USA)
}

\begin{abstract}
We examined international students' perceptions of the personal and professional value they receive from higher education in the United States. Results indicated that students' professional outcomes were significantly lower than their expectations related to their professional development, while students benefited personally to a much greater extent than they had anticipated. In this study, we identified areas of opportunities for higher education to support international students' professional and personal goals.
\end{abstract}

Keywords: value of higher education, international students, international students' engagement

The number of international students in the United States reached 820,000 in 2013, and it continues to grow despite increasing competition from Great Britain, Australia, and the sending countries (Alberts, 2007; Institute of International Education, 2013). Some research indicates that the decision to study in the United States is primarily driven by international students' expectation to improve their future career opportunities, and to obtain experience that will eventually lead to employment (Hazen \& Alberts, 2006). International education is expected to allow students to obtain a new perspective on their field of study, get a broader and more practice-oriented education, develop personally, and build intercultural friendships and networks (Obst \& Forster, 2007). These motivations are somewhat similar 
to those of U.S. students participating in study abroad programs, who often go abroad to master a foreign language, travel, and learn about a different culture. Despite some parallels, the experiences of U.S. students and international students do differ. Most study abroad programs are short-term, and they might create a somewhat "sheltered" environment, with the participants tending to remain connected to their U.S. peers and having a limited contact with the host country members (Allen, 2010).

Higher education greatly benefits not only culturally but also financially from international students' presence, but the support available to these students is not always sufficient or appropriate (Breuning, 2007). Language and cultural difficulties and the dearth of social support make it challenging to navigate U.S. institutions. For many international students, personal relationships with U.S. Americans, fluency in English and comfort using the language significantly impact the experience of U.S. higher education and positively contribute to their academic success and social adjustment (Yeh \& Inose, 2003). However, access to those relationships is not equal for all international students. Western Europeans who communicate well in English and who experience fewer cultural barriers are more likely to befriend U.S. Americans, interact with them more often, and consequently be better adjusted to the new environment (Trice, 2004).

To this end, our study examined the personal and professional value of U.S. higher education for international students. Specifically, the study explored what personal and professional goals influence the decision to study in the United States and the extent to which those goals are achieved, taking into account students' own engagement in goal achievement as well as institutional support.

\section{LITERATURE REVIEW}

Multiple studies report that international students encounter a variety of challenges caused by cultural, linguistic, social, and educational differences (Crockett and Hays, 2011; Olivas \& Li, 2006). The intensity of the problems related to cultural adjustment of international students depend on a variety of factors, such as students' English language ability, the perception of social acceptance, the length of time in the host country, students' race and ethnicity, and their experiences with discrimination (Constantine, Okazaki, \& Utsey, 2004; Yeh \& Inose, 2003). One of the challenges consistently reported in extant research is international students' difficulty with their social integration, which ultimately affects their learning and perception of success (Grayson, 2008; Yeh \& Inose, 2003). Although some of international students' difficulties are related to their personal backgrounds and experiences, host institutions can address many issues by 
providing appropriate support structures and facilitating an integration of international students in campus communities. In order for universities to identify specific barriers to international students' goal attainment, institutions need to better understand students' personal and professional goals, students' and institutional efforts in supporting these goals, and the extent to which these goals are attained.

Previous research reports a gap between international students' expectations of institutional support and their actual experiences. For instance, international students expect significant social support from university staff, including help with the development of social networks through formalized peer systems and social events; however, some university staff, particularly instructors, may perceive such expectations as exceeding their roles, and believe that students should be more self-reliant in regard to building their own social networks (Bartram, 2007). Many student service professionals serving international students are overwhelmed with the demands placed on them by immigration regulations and reporting requirements, leaving little time for providing counseling or advising, or creating programs that would alleviate adjustment difficulties (Rosser, Hermsen, Mamiseishvili, \& Wood, 2007). Despite significant needs expressed by international students, once they arrive at their campuses, they are less likely than U.S.-born students to seek assistance and opportunities for social interaction when faced with difficulties (Olivas \& $\mathrm{Li}$, 2006). Although career-related concerns are the most problematic, right after financial aid issues, the stress related to cultural adaptation makes it challenging to focus on career planning and development, consequently hindering international students' long-term career success (Galloway \& Jenkins, 2005; Reynolds \& Constantine, 2007).

Extant research has greatly contributed to our knowledge of the rewards and struggles international students undergo during their sojourn in the United States. Most previous studies focused on international students' transition into higher education, and some research addressed international students' expectations (Arthur, 2008; Chow, 2011; Sherry, Bhat, Beaver, \& Ling, 2004). Prior to this study no research could be found that had addressed international students' perceived value that studying in the United States adds to their personal and professional development, their expectations from higher education related to their professional and personal development and future career goals, and the extent to which international students are engaged in their own goal attainment. Therefore, our study posed the following research questions:

1. From international students' perspective, what value does U.S. higher education add to international students personally and professionally, specifically: 
a. what are international students' personal and professional goals for choosing to study in the United States;

b. to what extent are international students engaged in the achievement of their personal and professional goals;

c. what are international students' perceptions of the support they receive from higher education; and

d. how do international students perceive personal and professional value of their U.S. higher education experience?

2. What is the relationship between international students' goals and perceived benefits, and how is the relationship influenced by institutional support and international students' engagement in the achievement of their goals?

\section{RESEARCH METHOD}

A quantitative approach was utilized, where international students' perceptions were collected through a cross-sectional survey. This approach allowed for a numeric description of international students' opinions, attitudes, beliefs, and practices in regard to their personal and professional goals and the achievement of these goals, students' engagement in - and institutional support for - their goal attainment, as well as the relationship between students' goals and outcomes (Creswell, 2008). While qualitative research may have provided more detailed information on fewer international students, our goal was to obtain a broader set of data across a larger population.

This study was limited to a convenience sample of full-time bachelor, master, and doctoral international students at one public Midwestern higher education institution which recently expressed its strategic commitment to global engagement. Although this type of nonprobability sampling does not allow the researcher to state with confidence that the sample is representative of the population, it can offer valuable information to examine the research questions (Creswell, 2008). The target population for the study included 1,140 international students (567 undergraduate and 573 graduate) who were pursuing degrees at a university with an enrollment of approximately 24,000, with international students accounting for about $6.4 \%$. International students were defined as individuals born outside of the United States, who came to the United States to pursue higher education. The study focused on degree-seeking international students as these students have a long-term educational, social, and financial commitment to the institution and might have a different set of expectations from the host university and culture than non-degree seeking international students. The sampling frame, or the list of students meeting 
the criteria for the study, was obtained from the Office of Institutional Research at the university where the study was conducted. Given email access to the entire target population, all students in the sampling frame were invited to participate in the study.

While it would have been ideal to have found an existing survey with pre-established validity and reliability, none was found which matched our research questions. Thus we created our own survey based on an extensive literature review, including findings from Breuning (2007), Chow (2011), Ho, Bulman-Fleming, and Mitchell (2003), the National Survey of Student Engagement (2005), Obst and Forster (2007), Sherry et al. (2004), and Urban et al. (2010). The lack of established reliability and validity (beyond content validity) is viewed as a limitation to our results.

To enhance our response rate, the tailored design method was utilized (Dillman, Smyth, \& Christiam, 2009). The tailored design method, which is based on the social exchange theory, allows for the use of multiple motivational features to increase the quantity and the quality of responses. To this end, throughout the whole process, the researchers weigh a number of options, including the number, timing, mode, and visual design of contacts; type of incentives; and usage and type of additional materials. The social exchange theory encourages survey researchers to work toward increasing the benefits and decreasing the cost of participation while establishing trust (Dillman et al., 2009). An initial email invitation and two follow-up messages were sent to all potential participants with a description of the purpose of the study and a request to complete the online survey. Additional follow-up procedures included using the researcher's personal network of international students, contacting the leaders of relevant registered student organizations on campus, and contacting staff in the international student office to encourage survey completion. Participation in the research was voluntary and confidential. The survey instrument included Likert-type scale questions (Creswell, 2008) in the following content areas: (a) international students' personal and professional reasons for choosing to study in the United States, (b) perceived benefits of studying at a U.S. higher education institution, (c) international students' engagement in the achievement of their personal and professional goals, and (d) international students' perceptions of the assistance received from higher education in support of their personal and professional goals.

Before survey administration, the instrument was pilot-tested in order to establish content validity, improve the survey format, questions, and scales, and estimate the average time it would take respondents to complete the survey (Creswell, 2008). To conduct pilot testing, a hard copy of the survey was given to five international students, who also completed the survey online. Data from pilot testing were not used for the study. The 
individuals who participated in pilot testing were asked to write their comments on the hard copy of the survey, which was submitted to the researcher. The comments were evaluated by the researcher, and necessary changes were incorporated to finalize the instrument. The full instrument can be found within Urban (2012).

For each of the statements in the survey, descriptive statistics including frequencies, means and standard deviations were calculated to display respondents' ratings on the importance of various issues. Cronbach's alpha was used to measure the internal consistency due to collapsing the data (Creswell, 2008). To understand the relationships between key variables, multiple regression and paired sample t-tests were used (Gall, Gall, \& Borg, 2007).

\section{RESULTS}

\section{Respondents}

Responses were obtained from 249 students, representing 22\% of the target population. Over half of respondents were male (52.6\%), 40.6\% were female, and $.8 \%$ were transgender; $53.8 \%$ were graduate students and 41.7\% were undergraduates. Participants' age ranged from 18 to 62, with the mean of 25.85 ( $S D=6.14)$; more than half of the participants $(64.5 \%)$ were between 18 and 26 years old. Respondents had been in the United States between 1 and 16 years, with $81 \%$ having been in the United States between one and four years $(M=2.82 ; S D=2.19)$. Respondents' length of time at this university ranged from 1 to 10 years, with $86 \%$ having been at the institution between one and four years $(M=2.35$; $S D=1.57)$.

The regions of origin in the sample represent the regions from which the target population was drawn, with $20.1 \%$ of respondents from South and Central America, 19.7\% from East Asia, 13.3\% from South and Central Asia, 12.4\% from the Middle East, 11.2\% from Africa, 8.4\% from Southeast Asia, 5.6\% from Europe, and 1.2\% from North America. Participants' areas of study included engineering (29.3\%), business (18.9\%), sciences $(14.1 \%)$, education (11.6\%), humanities $(8.8 \%)$, social sciences (7.6\%), fine arts (2.4\%), and healthcare (1.2\%).

\section{Value of U.S. Higher Education}

The top three motivators for coming to the United States were related to students' professional development, including getting a good quality education $(M=4.55, S D=.71)$, learning new ways of thinking and acting in the field of study $(M=4.30, S D=.81)$, and getting practical experience $(M=4.27, S D=.88)$. The three least important reasons were related to personal development and included learning to adjust to new 
Table 1: Rank Ordering of Reasons for Studying in the U.S. $(N=249)$

\begin{tabular}{lcccccc}
\hline I decided to study in & 1 & 2 & 3 & 4 & 5 & Mean \\
the U.S. to: & $n(\%)$ & $n(\%)$ & $n(\%)$ & $n(\%)$ & $n(\%)$ & $(S D)$ \\
\hline Get good quality & 1 & 3 & 18 & 64 & 163 & 4.55 \\
education & $(0.4)$ & $(1.2)$ & $(7.2)$ & $(25.7)$ & $(65.5)$ & $(0.71)$ \\
Learn new ways of & 2 & 5 & 28 & 92 & 119 & 4.30 \\
thinking and & $(0.8)$ & $(2.0)$ & $(11.2)$ & $(36.9)$ & $(47.8)$ & $(0.81)$ \\
acting in my field & & & & & & \\
Get practical & 4 & 3 & 38 & 80 & 122 & 4.27 \\
experience & $(1.6)$ & $(1.2)$ & $(15.3)$ & $(33.1)$ & $(49.0)$ & $(0.88)$ \\
Develop skills to get & 3 & 10 & 36 & 84 & 116 & 4.20 \\
a good job & $(1.2)$ & $(4.0)$ & $(14.5)$ & $(33.7)$ & $(46.6)$ & $(0.91)$ \\
Get practice- & 2 & 14 & 37 & 82 & 111 & 4.16 \\
oriented education & $(0.8)$ & $(5.6)$ & $(14.9)$ & $(33.9)$ & $(44.6)$ & $(0.93)$ \\
Learn to work in a & 4 & 15 & 35 & 86 & 109 & 4.13 \\
cross-cultural & $(1.6)$ & $(6.0)$ & $(14.1)$ & $(34.5)$ & $(43.8)$ & $(0.97)$ \\
environment & & & & & & \\
Meet professionals & 4 & 19 & 45 & 75 & 101 & 4.02 \\
in my field & $(1.6)$ & $(7.6)$ & $(18.1)$ & $(30.1)$ & $(40.6)$ & $(1.03)$ \\
Become more & 12 & 22 & 40 & 59 & 114 & 3.98 \\
independent & $(4.8)$ & $(8.8)$ & $(16.1)$ & $(23.7)$ & $(45.8)$ & $(1.19)$ \\
Improve English & 20 & 23 & 37 & 57 & 112 & 3.88 \\
& $(8.0)$ & $(9.2)$ & $(14.9)$ & $(22.9)$ & $(45.0)$ & $(1.29)$ \\
Learn about & 8 & 29 & 53 & 78 & 80 & 3.78 \\
different cultural & $(3.2)$ & $(11.6)$ & $(21.3)$ & $(31.3)$ & $(32.1)$ & $(1.11)$ \\
viewpoints & & & & & & \\
Build intercultural & 14 & 23 & 59 & 79 & 74 & 3.71 \\
friendships & $(5.6)$ & $(9.2)$ & $(23.7)$ & $(31.7)$ & $(29.7)$ & $(1.15)$ \\
Learn to adjust to & 13 & 24 & 69 & 75 & 66 & 3.64 \\
new social and & $(5.2)$ & $(9.6)$ & $(27.7)$ & $(30.1)$ & $(26.5)$ & $(1.13)$ \\
cultural customs & & & & & & \\
Understand myself & 23 & 21 & 65 & 65 & 72 & 3.58 \\
better & $(9.2)$ & $(8.4)$ & $(26.1)$ & $(26.1)$ & $(28.9)$ & $(1.25)$ \\
Keep my family & 92 & 37 & 50 & 27 & 41 & 2.55 \\
happy & $(36.9)$ & $(14.9)$ & $(20.1)$ & $(10.8)$ & $(16.5)$ & $(1.48)$ \\
\hline
\end{tabular}

Likert scale: Not at all a reason=1, Not a very important reason=2, Somewhat important reason=3, Very important reason=4, Extremely important reason=5 
Table 2: Rank Ordering of Students' Engagement in Goal Achievement $(N=249)$

\begin{tabular}{|c|c|c|c|c|c|c|}
\hline $\begin{array}{l}\text { At this university, how } \\
\text { often have you: }\end{array}$ & $\begin{array}{c}1 \\
\mathrm{n} \\
(\%)\end{array}$ & $\begin{array}{c}2 \\
\mathrm{n} \\
(\%)\end{array}$ & $\begin{array}{c}3 \\
\mathrm{n} \\
(\%)\end{array}$ & $\begin{array}{c}4 \\
\mathrm{n} \\
(\%)\end{array}$ & $\begin{array}{c}5 \\
\mathrm{n} \\
(\%)\end{array}$ & $\begin{array}{l}\text { Mean } \\
\text { (SD) }\end{array}$ \\
\hline $\begin{array}{l}\text { Made friends with other } \\
\text { intl students outside } \\
\text { of class }\end{array}$ & $\begin{array}{c}6 \\
(2.4)\end{array}$ & $\begin{array}{c}18 \\
(7.2)\end{array}$ & $\begin{array}{c}69 \\
(27.7)\end{array}$ & $\begin{array}{c}81 \\
(32.5)\end{array}$ & $\begin{array}{c}70 \\
(28.1)\end{array}$ & $\begin{array}{c}3.78 \\
(1.02)\end{array}$ \\
\hline $\begin{array}{l}\text { Asked questions in } \\
\text { class or contributed to } \\
\text { discussions }\end{array}$ & $\begin{array}{c}2 \\
(0.8)\end{array}$ & $\begin{array}{c}32 \\
(12.9)\end{array}$ & $\begin{array}{c}77 \\
(30.9)\end{array}$ & $\begin{array}{c}78 \\
(31.3)\end{array}$ & $\begin{array}{c}55 \\
(22.1)\end{array}$ & $\begin{array}{l}3.62 \\
(1.0)\end{array}$ \\
\hline $\begin{array}{l}\text { Made friends with U.S. } \\
\text { students outside of } \\
\text { class }\end{array}$ & $\begin{array}{c}14 \\
(5.6)\end{array}$ & $\begin{array}{c}29 \\
(11.6)\end{array}$ & $\begin{array}{c}81 \\
(32.5)\end{array}$ & $\begin{array}{c}64 \\
(25.7)\end{array}$ & $\begin{array}{c}58 \\
(23.3)\end{array}$ & $\begin{array}{c}3.50 \\
(1.14)\end{array}$ \\
\hline $\begin{array}{l}\text { Had serious } \\
\text { conversations with } \\
\text { students different } \\
\text { from you }\end{array}$ & $\begin{array}{c}20 \\
(8.0)\end{array}$ & $\begin{array}{c}40 \\
(16.1)\end{array}$ & $\begin{array}{c}84 \\
(33.7)\end{array}$ & $\begin{array}{c}59 \\
(23.7)\end{array}$ & $\begin{array}{c}43 \\
(17.3)\end{array}$ & $\begin{array}{c}3.26 \\
(1.16)\end{array}$ \\
\hline $\begin{array}{l}\text { Talked about career } \\
\text { plans with a } \\
\text { prof/advisor }\end{array}$ & $\begin{array}{c}28 \\
(11.2)\end{array}$ & $\begin{array}{c}47 \\
(18.9)\end{array}$ & $\begin{array}{c}85 \\
(34.1)\end{array}$ & $\begin{array}{c}55 \\
(22.1)\end{array}$ & $\begin{array}{c}29 \\
(11.6)\end{array}$ & $\begin{array}{c}3.04 \\
(1.16)\end{array}$ \\
\hline $\begin{array}{l}\text { Developed relationships } \\
\text { with people outside } \\
\text { the university }\end{array}$ & $\begin{array}{c}36 \\
(14.5)\end{array}$ & $\begin{array}{c}49 \\
(19.7)\end{array}$ & $\begin{array}{c}79 \\
(31.7)\end{array}$ & $\begin{array}{c}45 \\
(18.1)\end{array}$ & $\begin{array}{c}37 \\
(14.9)\end{array}$ & $\begin{array}{c}2.99 \\
(1.25)\end{array}$ \\
\hline $\begin{array}{l}\text { Asked profs/advisors } \\
\text { for help with } \\
\text { academic or language } \\
\text { difficulties }\end{array}$ & $\begin{array}{c}47 \\
(18.9)\end{array}$ & $\begin{array}{c}52 \\
(20.9)\end{array}$ & $\begin{array}{c}75 \\
(30.1)\end{array}$ & $\begin{array}{c}54 \\
(21.7)\end{array}$ & $\begin{array}{c}18 \\
(7.2)\end{array}$ & $\begin{array}{l}2.77 \\
(1.2)\end{array}$ \\
\hline Used student services & $\begin{array}{c}50 \\
(20.1)\end{array}$ & $\begin{array}{c}56 \\
(22.5)\end{array}$ & $\begin{array}{c}66 \\
(26.5)\end{array}$ & $\begin{array}{c}51 \\
(20.5)\end{array}$ & $\begin{array}{c}21 \\
(8.4)\end{array}$ & $\begin{array}{c}2.74 \\
(1.24)\end{array}$ \\
\hline $\begin{array}{l}\text { Got involved in student } \\
\text { org made up mostly } \\
\text { of intl students }\end{array}$ & $\begin{array}{c}64 \\
(25.7)\end{array}$ & $\begin{array}{c}53 \\
(21.3)\end{array}$ & $\begin{array}{c}54 \\
(21.7)\end{array}$ & $\begin{array}{c}39 \\
(15.7)\end{array}$ & $\begin{array}{c}36 \\
(14.5)\end{array}$ & $\begin{array}{c}2.72 \\
(1.38)\end{array}$ \\
\hline Used career services & $\begin{array}{c}56 \\
(22.5)\end{array}$ & $\begin{array}{c}59 \\
(23.7)\end{array}$ & $\begin{array}{c}75 \\
(30.1)\end{array}$ & $\begin{array}{c}29 \\
(11.6)\end{array}$ & $\begin{array}{c}22 \\
(8.8)\end{array}$ & $\begin{array}{c}2.59 \\
(1.22)\end{array}$ \\
\hline $\begin{array}{l}\text { Asked U.S. students for } \\
\text { help with academic or } \\
\text { language difficulties }\end{array}$ & $\begin{array}{c}65 \\
(26.1)\end{array}$ & $\begin{array}{c}51 \\
(20.5)\end{array}$ & $\begin{array}{c}68 \\
(27.3)\end{array}$ & $\begin{array}{c}41 \\
(16.5)\end{array}$ & $\begin{array}{c}20 \\
(8.0)\end{array}$ & $\begin{array}{c}2.59 \\
(1.26)\end{array}$ \\
\hline $\begin{array}{l}\text { Asked intl students for } \\
\text { help with academic or } \\
\text { language difficulties }\end{array}$ & $\begin{array}{c}69 \\
(27.7)\end{array}$ & $\begin{array}{c}56 \\
(22.5)\end{array}$ & $\begin{array}{c}59 \\
(23.7)\end{array}$ & $\begin{array}{c}42 \\
(16.9)\end{array}$ & $\begin{array}{c}20 \\
(8.0)\end{array}$ & $\begin{array}{c}2.54 \\
(1.28)\end{array}$ \\
\hline $\begin{array}{l}\text { Got involved in student } \\
\text { org made up mostly }\end{array}$ & $\begin{array}{c}91 \\
(36.5)\end{array}$ & $\begin{array}{c}55 \\
(22.1)\end{array}$ & $\begin{array}{c}50 \\
(20.1)\end{array}$ & $\begin{array}{c}22 \\
(8.8)\end{array}$ & $\begin{array}{c}26 \\
(10.4)\end{array}$ & $\begin{array}{c}2.33 \\
(1.34)\end{array}$ \\
\hline
\end{tabular}


social and cultural customs $(M=3.64, S D=1.13)$, understanding oneself better $(M=3.58, S D=1.25)$, and keeping family happy $(M=2.55, S D=$ 1.48). Table 1 provides rank ordering of the means for the reasons for studying in the United States as reported by participants.

As far as students' engagement in goal achievement, the most frequent activities included making friends with other international students outside of class $(M=3.78, S D=1.02)$, asking questions in class or contributing to class discussions $(M=3.63, S D=1.0)$, and making friends with U.S. students outside of class $(M=3.50, S D=1.14)$. The least frequent activities were asking U.S. students for help with academic or language difficulties $(M=2.59, S D=1.26)$, asking other international students for help with academic or language difficulties $(M=2.54, S D=$ 1.28), and getting involved in an organization made up mostly of U.S. students $(M=2.33, S D=1.34)$. Table 2 provides frequencies and means for all items related to students' engagement in goal achievement.

In regard to the level of agreement on items related to institutional support and environment, students largely agreed that it is easy to make friends with other international students $(M=4.82, S D=.97)$, professors and staff are willing to help international students with academic difficulties $(M=4.76, S D=1.11)$, and students are taught the skills for employment ( $M$ $=4.63, S D=1.08)$. Respondents agreed the least that it is easy to make friends with U.S. students $(M=4.17, S D=1.28)$, professors and staff understand the needs of international students $(M=4.15, S D=1.30)$, and they are encouraged to participate in co-curricular activities $(M=4.08, S D=$ 1.27). Table 3 offers rank ordering of students' perceptions of institutional assistance for goal achievement.

In regard to the perceived benefit from U.S. higher education, the top areas included getting quality education $(M=4.21, S D=.82)$, becoming more independent $(M=4.7, S D=1.11)$, and learning new ways of thinking and acting in the field of study $(M=4.6, S D=.90)$. The lowest rated aspects were writing clearly and effectively $(M=3.76, S D=.96)$, understanding themselves better $(M=3.72, S D=1.13)$, and getting practical experience $(M=3.69, S D=1.17)$. Table 4 contains the rank ordering of students' perceptions of the value received from U.S. higher education.

\section{Relationship Between Goals and Perceived Benefits}

To identify the relationship between students' goals and perceived benefits, paired-samples t-tests were performed for the items which asked participants about the reasons they came to the United States with matching items asking about the extent to which students' goals have been met. International students' outcomes related to their professional development 


\section{Table 3: Rank Ordering of Students' Perception of Institutional}

Support $(N=246)$

\begin{tabular}{|c|c|c|c|c|c|c|c|}
\hline At this university: & $\begin{array}{c}1 \\
n \\
(\%)\end{array}$ & $\begin{array}{c}2 \\
n \\
(\%)\end{array}$ & $\begin{array}{c}3 \\
n(\%)\end{array}$ & $\begin{array}{c}4 \\
n(\%)\end{array}$ & $\begin{array}{c}5 \\
n(\%)\end{array}$ & $\begin{array}{c}6 \\
n(\%)\end{array}$ & $\begin{array}{l}\text { Mean } \\
(S D)\end{array}$ \\
\hline $\begin{array}{l}\text { It is easy to make } \\
\text { friends with intl } \\
\text { students }\end{array}$ & $\begin{array}{c}0 \\
(0.0)\end{array}$ & $\begin{array}{c}4 \\
(1.6)\end{array}$ & $\begin{array}{c}17 \\
(6.8)\end{array}$ & $\begin{array}{c}65 \\
(26.1)\end{array}$ & $\begin{array}{c}89 \\
(35.7)\end{array}$ & $\begin{array}{c}68 \\
(27.3)\end{array}$ & $\begin{array}{c}4.82 \\
(0.97)\end{array}$ \\
\hline $\begin{array}{l}\text { Profs and staff willing } \\
\text { to help intl students } \\
\text { with academic } \\
\text { difficulties }\end{array}$ & $\begin{array}{c}4 \\
(1.6)\end{array}$ & $\begin{array}{c}7 \\
(2.8)\end{array}$ & $\begin{array}{c}18 \\
(7.2)\end{array}$ & $\begin{array}{c}54 \\
(21.7)\end{array}$ & $\begin{array}{c}95 \\
(38.2)\end{array}$ & $\begin{array}{c}67 \\
(26.9)\end{array}$ & $\begin{array}{c}4.76 \\
(1.11)\end{array}$ \\
\hline $\begin{array}{l}\text { I am taught skills I } \\
\text { need for employment }\end{array}$ & $\begin{array}{c}5 \\
(2.0)\end{array}$ & $\begin{array}{c}5 \\
(2.0)\end{array}$ & $\begin{array}{c}16 \\
(6.4)\end{array}$ & $\begin{array}{c}78 \\
(31.3)\end{array}$ & $\begin{array}{c}88 \\
(35.3)\end{array}$ & $\begin{array}{c}54 \\
(21.7)\end{array}$ & $\begin{array}{c}4.63 \\
(1.08)\end{array}$ \\
\hline $\begin{array}{l}\text { It is easy to develop } \\
\text { relationships with } \\
\text { profs and staff }\end{array}$ & $\begin{array}{c}1 \\
(0.4)\end{array}$ & $\begin{array}{c}7 \\
(2.8)\end{array}$ & $\begin{array}{c}22 \\
(8.8)\end{array}$ & $\begin{array}{c}75 \\
(30.1)\end{array}$ & $\begin{array}{c}87 \\
(34.9)\end{array}$ & $\begin{array}{c}52 \\
(20.9)\end{array}$ & $\begin{array}{c}4.62 \\
(1.03)\end{array}$ \\
\hline $\begin{array}{l}\text { Profs and staff willing } \\
\text { to help intl students } \\
\text { with cross-cultural } \\
\text { com }\end{array}$ & $\begin{array}{c}1 \\
(0.4)\end{array}$ & $\begin{array}{c}13 \\
(5.2)\end{array}$ & $\begin{array}{c}27 \\
(10.8)\end{array}$ & $\begin{array}{c}65 \\
(26.1)\end{array}$ & $\begin{array}{c}90 \\
(36.1)\end{array}$ & $\begin{array}{c}50 \\
(20.1)\end{array}$ & $\begin{array}{c}4.54 \\
(1.11)\end{array}$ \\
\hline $\begin{array}{l}\text { I have opportunities to } \\
\text { practically apply my } \\
\text { knowledge }\end{array}$ & $\begin{array}{c}4 \\
(1.6)\end{array}$ & $\begin{array}{c}11 \\
(4.4)\end{array}$ & $\begin{array}{c}20 \\
(8.0)\end{array}$ & $\begin{array}{c}85 \\
(34.1)\end{array}$ & $\begin{array}{c}77 \\
(30.9)\end{array}$ & $\begin{array}{c}48 \\
(19.3)\end{array}$ & $\begin{array}{c}4.49 \\
(1.12)\end{array}$ \\
\hline $\begin{array}{l}\text { Profs and staff } \\
\text { encourage contact } \\
\text { among students from } \\
\text { different } \\
\text { backgrounds }\end{array}$ & $\begin{array}{c}5 \\
(2.0)\end{array}$ & $\begin{array}{c}21 \\
(8.4)\end{array}$ & $\begin{array}{c}33 \\
(13.3)\end{array}$ & $\begin{array}{c}63 \\
(25.3)\end{array}$ & $\begin{array}{c}85 \\
(34.1)\end{array}$ & $\begin{array}{c}37 \\
(14.9)\end{array}$ & $\begin{array}{c}4.28 \\
(1.24)\end{array}$ \\
\hline $\begin{array}{l}\text { Other students are } \\
\text { willing to help intl } \\
\text { students }\end{array}$ & $\begin{array}{c}6 \\
(2.4)\end{array}$ & $\begin{array}{c}17 \\
(6.8)\end{array}$ & $\begin{array}{c}43 \\
(17.3)\end{array}$ & $\begin{array}{c}71 \\
(28.5)\end{array}$ & $\begin{array}{c}76 \\
(30.5)\end{array}$ & $\begin{array}{c}33 \\
(13.3)\end{array}$ & $\begin{array}{c}4.19 \\
(1.21)\end{array}$ \\
\hline $\begin{array}{l}\text { It is easy to make } \\
\text { friends with U.S. } \\
\text { students }\end{array}$ & $\begin{array}{c}9 \\
(3.6)\end{array}$ & $\begin{array}{c}19 \\
(7.6)\end{array}$ & $\begin{array}{c}36 \\
(14.5)\end{array}$ & $\begin{array}{c}73 \\
(29.3)\end{array}$ & $\begin{array}{c}71 \\
(28.5)\end{array}$ & $\begin{array}{c}36 \\
(14.5)\end{array}$ & $\begin{array}{c}4.17 \\
(1.28)\end{array}$ \\
\hline $\begin{array}{l}\text { Profs and staff } \\
\text { understand the needs } \\
\text { of intl students }\end{array}$ & $\begin{array}{c}10 \\
(4.0)\end{array}$ & $\begin{array}{c}20 \\
(8.0)\end{array}$ & $\begin{array}{c}34 \\
(13.7)\end{array}$ & $\begin{array}{c}76 \\
(30.5)\end{array}$ & $\begin{array}{c}68 \\
(27.3)\end{array}$ & $\begin{array}{c}36 \\
(14.5)\end{array}$ & $\begin{array}{c}4.15 \\
(1.30)\end{array}$ \\
\hline $\begin{array}{l}\text { I am encouraged to } \\
\text { participate in co- } \\
\text { curricular activities }\end{array}$ & $\begin{array}{c}8 \\
(3.2)\end{array}$ & $\begin{array}{c}23 \\
(9.2)\end{array}$ & $\begin{array}{c}39 \\
(15.7)\end{array}$ & $\begin{array}{c}77 \\
(30.9)\end{array}$ & $\begin{array}{c}65 \\
(26.1)\end{array}$ & $\begin{array}{c}32 \\
(12.9)\end{array}$ & $\begin{array}{c}4.08 \\
(1.27)\end{array}$ \\
\hline
\end{tabular}

Likert scale: Strongly Disagree=1, Disagree=2, Moderately Disagree=3, Moderately Agree $=4$, Agree $=5$, Strongly Agree $=6$ 
Table 4: Rank Ordering of Students' Perceptions of the Value of Higher Education $(N=248)$

\begin{tabular}{|c|c|c|c|c|c|c|}
\hline $\begin{array}{l}\text { To what extent have you } \\
\text { accomplished: }\end{array}$ & $\begin{array}{c}1 \\
n \\
(\%)\end{array}$ & $\begin{array}{c}2 \\
n \\
(\%)\end{array}$ & $\begin{array}{c}3 \\
n \\
(\%)\end{array}$ & $\begin{array}{c}4 \\
n \\
(\%)\end{array}$ & $\begin{array}{c}5 \\
n \\
(\%)\end{array}$ & $\begin{array}{l}\text { Mean } \\
(S D)\end{array}$ \\
\hline $\begin{array}{l}\text { Get good quality } \\
\text { education }\end{array}$ & $\begin{array}{c}1 \\
(0.4)\end{array}$ & $\begin{array}{c}6 \\
(2.4)\end{array}$ & $\begin{array}{c}40 \\
(16.1)\end{array}$ & $\begin{array}{c}93 \\
(37.3)\end{array}$ & $\begin{array}{c}108 \\
(43.4)\end{array}$ & $\begin{array}{c}4.21 \\
(0.82)\end{array}$ \\
\hline $\begin{array}{l}\text { Become more } \\
\text { independent }\end{array}$ & $\begin{array}{c}15 \\
(6.0)\end{array}$ & $\begin{array}{c}5 \\
(2.0)\end{array}$ & $\begin{array}{c}39 \\
(15.7)\end{array}$ & $\begin{array}{c}75 \\
(30.1)\end{array}$ & $\begin{array}{c}112 \\
(45.0)\end{array}$ & $\begin{array}{c}4.07 \\
(1.11)\end{array}$ \\
\hline $\begin{array}{l}\text { Learn new ways of } \\
\text { thinking \& acting in } \\
\text { field }\end{array}$ & $\begin{array}{c}1 \\
(0.4)\end{array}$ & $\begin{array}{c}15 \\
(6.0)\end{array}$ & $\begin{array}{c}43 \\
(17.3)\end{array}$ & $\begin{array}{c}96 \\
(38.6)\end{array}$ & $\begin{array}{c}92 \\
(36.9)\end{array}$ & $\begin{array}{c}4.06 \\
(0.90)\end{array}$ \\
\hline $\begin{array}{l}\text { Think critically and } \\
\text { analytically }\end{array}$ & $\begin{array}{c}3 \\
(1.2)\end{array}$ & $\begin{array}{c}15 \\
(6.0)\end{array}$ & $\begin{array}{c}41 \\
(16.5)\end{array}$ & $\begin{array}{c}102 \\
(41.0)\end{array}$ & $\begin{array}{c}84 \\
(33.7)\end{array}$ & $\begin{array}{l}4.02 \\
(.93)\end{array}$ \\
\hline $\begin{array}{l}\text { Learn to work in a cross- } \\
\text { cultural environment }\end{array}$ & $\begin{array}{c}2 \\
(0.8)\end{array}$ & $\begin{array}{c}18 \\
(7.2)\end{array}$ & $\begin{array}{c}50 \\
(20.1)\end{array}$ & $\begin{array}{c}97 \\
(39.0)\end{array}$ & $\begin{array}{c}80 \\
(32.1)\end{array}$ & $\begin{array}{c}3.95 \\
(0.94)\end{array}$ \\
\hline $\begin{array}{l}\text { Build intercultural } \\
\text { friendships }\end{array}$ & $\begin{array}{c}10 \\
(4.0)\end{array}$ & $\begin{array}{c}12 \\
(4.8)\end{array}$ & $\begin{array}{c}49 \\
(19.7)\end{array}$ & $\begin{array}{c}87 \\
(34.9)\end{array}$ & $\begin{array}{c}88 \\
(35.3)\end{array}$ & $\begin{array}{c}3.94 \\
(1.05)\end{array}$ \\
\hline $\begin{array}{l}\text { Get practice-oriented } \\
\text { education }\end{array}$ & $\begin{array}{c}6 \\
(2.4)\end{array}$ & $\begin{array}{l}19 \\
(7.6)\end{array}$ & $\begin{array}{c}56 \\
(22.5)\end{array}$ & $\begin{array}{c}75 \\
(30.1)\end{array}$ & $\begin{array}{c}90 \\
(36.1)\end{array}$ & $\begin{array}{c}3.92 \\
(1.05)\end{array}$ \\
\hline Improve English & $\begin{array}{c}12 \\
(4.8)\end{array}$ & $\begin{array}{l}18 \\
(7.2)\end{array}$ & $\begin{array}{c}41 \\
(16.5)\end{array}$ & $\begin{array}{c}83 \\
(33.3)\end{array}$ & $\begin{array}{c}93 \\
(37.3)\end{array}$ & $\begin{array}{c}3.92 \\
(1.12)\end{array}$ \\
\hline $\begin{array}{l}\text { Learn about different } \\
\text { cultural viewpoints }\end{array}$ & $\begin{array}{c}8 \\
(3.2)\end{array}$ & $\begin{array}{c}23 \\
(9.2)\end{array}$ & $\begin{array}{c}40 \\
(16.1)\end{array}$ & $\begin{array}{c}94 \\
(37.8)\end{array}$ & $\begin{array}{c}79 \\
(31.7)\end{array}$ & $\begin{array}{c}3.87 \\
(1.07)\end{array}$ \\
\hline $\begin{array}{l}\text { Develop work-related } \\
\text { knowledge and skills }\end{array}$ & $\begin{array}{c}6 \\
(2.5)\end{array}$ & $\begin{array}{c}19 \\
(7.6)\end{array}$ & $\begin{array}{c}58 \\
(23.3)\end{array}$ & $\begin{array}{c}88 \\
(35.3)\end{array}$ & $\begin{array}{c}76 \\
(30.5)\end{array}$ & $\begin{array}{c}3.85 \\
(1.02)\end{array}$ \\
\hline $\begin{array}{l}\text { Speak clearly and } \\
\text { effectively }\end{array}$ & $\begin{array}{c}4 \\
(1.6)\end{array}$ & $\begin{array}{c}19 \\
(7.6)\end{array}$ & $\begin{array}{c}58 \\
(23.3)\end{array}$ & $\begin{array}{c}98 \\
(39.4)\end{array}$ & $\begin{array}{c}68 \\
(27.3)\end{array}$ & $\begin{array}{c}3.84 \\
(0.97)\end{array}$ \\
\hline $\begin{array}{l}\text { Adjust to new social and } \\
\text { cultural customs }\end{array}$ & $\begin{array}{c}12 \\
(4.8)\end{array}$ & $\begin{array}{c}14 \\
(5.6)\end{array}$ & $\begin{array}{c}55 \\
(22.1)\end{array}$ & $\begin{array}{c}88 \\
(35.3)\end{array}$ & $\begin{array}{c}77 \\
(30.9)\end{array}$ & $\begin{array}{c}3.83 \\
(1.08)\end{array}$ \\
\hline $\begin{array}{l}\text { Meet professionals in my } \\
\text { field }\end{array}$ & $\begin{array}{c}6 \\
(2.4)\end{array}$ & $\begin{array}{c}27 \\
(10.8)\end{array}$ & $\begin{array}{c}59 \\
(23.7)\end{array}$ & $\begin{array}{c}81 \\
(32.5)\end{array}$ & $\begin{array}{c}73 \\
(29.3)\end{array}$ & $\begin{array}{c}3.76 \\
(1.07)\end{array}$ \\
\hline $\begin{array}{l}\text { Write clearly and } \\
\text { effectively }\end{array}$ & $\begin{array}{c}3 \\
(1.2)\end{array}$ & $\begin{array}{c}25 \\
(10.0)\end{array}$ & $\begin{array}{c}58 \\
(23.3)\end{array}$ & $\begin{array}{c}103 \\
(41.4)\end{array}$ & $\begin{array}{c}58 \\
(23.3)\end{array}$ & $\begin{array}{c}3.76 \\
(0.96)\end{array}$ \\
\hline Understand myself better & $\begin{array}{l}15 \\
(6.0)\end{array}$ & $\begin{array}{c}16 \\
(6.4)\end{array}$ & $\begin{array}{c}63 \\
(25.3)\end{array}$ & $\begin{array}{c}81 \\
(32.5)\end{array}$ & $\begin{array}{c}72 \\
(28.9)\end{array}$ & $\begin{array}{c}3.72 \\
(1.13)\end{array}$ \\
\hline Get practical experience & $\begin{array}{l}12 \\
(4.8)\end{array}$ & $\begin{array}{c}34 \\
(13.7)\end{array}$ & $\begin{array}{c}46 \\
(18.5)\end{array}$ & $\begin{array}{c}83 \\
(33.3)\end{array}$ & $\begin{array}{c}73 \\
(29.3)\end{array}$ & $\begin{array}{c}3.69 \\
(1.17)\end{array}$ \\
\hline
\end{tabular}

Likert scale: Not at all helped $=1$, Helped to a little extent $=2$, Helped to a moderate extent $=3$, Helped to a great extent $=4$, Helped to a very great extent $=5$. 
were much lower than respective professional goals, and the difference was statistically significant in all seven areas examined (see Figure 1). Students' personal outcomes were higher than their respective personal goals, which suggest that the experience of studying in the United States has been more personally enriching that the students had expected it to be. The difference between the three of these personal goals and respective outcomes was statistically significant.

\section{Figure 1: Relationship between professional and personal goals and outcomes.}

\begin{tabular}{lcc|c}
\hline \multicolumn{1}{c}{$\begin{array}{c}\text { PROFESSIONAL GOALS AND } \\
\text { OUTCOMES }\end{array}$} & $\begin{array}{c}\text { Goal } \\
\text { Mea }\end{array}$ & $\begin{array}{c}\text { Outco } \\
\text { me } \\
\text { Mean }\end{array}$ & $\begin{array}{c}\text { Mean } \\
\text { Diff. }\end{array}$ \\
& $\uparrow$ & $\downarrow$ & \\
\hline Get practical experience* & 4.27 & 3.69 & $-.58^{*}$ \\
Develop skills to get a good job* & 4.20 & 3.85 & $-.35^{*}$ \\
Get quality education* & 4.55 & 4.21 & $-.34^{*}$ \\
Meet professionals* & 4.02 & 3.76 & $-.25^{*}$ \\
Get practice-oriented education* & 4.16 & 3.92 & $-.24^{*}$ \\
Learn new ways of thinking and acting in field & 4.30 & 4.06 & $-.24^{*}$ \\
of study* & & & \\
Learn to work in cross-cultural environment* & 4.13 & 3.95 & $-.17^{*}$ \\
\hline & Goal & Outco & Mean \\
$\quad$ PERSONAL GOALS AND OUTCOMES & Mea & me & Diff. \\
& $\mathrm{n}$ & Mean & \\
& $\downarrow$ & $\uparrow$ & \\
\hline Build intercultural friendships* & 3.71 & 3.94 & $.23^{*}$ \\
Learn to adjust to new social and cultural & 3.64 & 3.83 & $.18^{*}$ \\
customs & & & \\
Understand myself better* & 3.58 & 3.72 & $.14^{*}$ \\
Learn about different cultural viewpoints & 3.78 & 3.87 & .09 \\
Become more independent & 3.98 & 4.07 & .09 \\
Improve English & 3.88 & 3.92 & .04 \\
\hline
\end{tabular}

$*$ Difference is significant at $\mathrm{p}<0.05$

Multiple regression was conducted to examine the extent to which international students' perception of the value from higher education is related to a combination of variables including their goals, institutional support, and their own engagement in goal achievement. We created six new collapsed variables: international students' goals, engagement in goal 
achievement, institutional support, value related to professional outcomes, value related to personal outcomes, and overall value of education. International students' goals, institutional support, and engagement in goal achievement explain $43 \%$ of variability in international students' perception of the overall value of higher education.

Further analyses revealed that three items within the category of international students' goals explained $34 \%$ of variability in perceived value, including: (1) learning to adjust to new social and cultural customs, (2) becoming more independent, and (3) getting a good quality education. Within the institutional support category, $26 \%$ of variability in perceived value was explained by three items: (1) I am taught skills for employment, (2) professors and staff encourage contact among students from different backgrounds, and (3) it is easy to make friends with other international students. Within the area of students' engagement in goal achievement, $18 \%$ of variability in perceived value can be explained by students (1) talking about career plans with professors or advisors, (2) having serious conversations with students different from oneself, and (3) using career services.

The examination of how specific aspects of students' own engagement in goal attainment and university support impact the value related to professional and personal outcomes revealed that $34 \%$ of the variability in value related to professional outcomes can be explained by (1) having opportunities to practically apply academic knowledge, (2) being taught skills needed for future employment, (3) talking about career plans with professors or advisors, and (4) using career services. Similarly, 23\% of the variability in value related to personal outcomes is accounted for by: (1) having professors and staff encourage contact among students from different backgrounds, (2) using career services, (3) other students' willingness to help international students, (4) having serious conversations with students different from oneself, and (5) using student services.

Figure 2 offers an overall summary of the items that are predictive of international students' perceived overall value of U.S. higher education and value related to personal and professional outcomes, as well as the items that were not predictive of such outcomes. When conducting multiple comparisons, adopting a strategy of adjusting the $p$-value downward for fear of committing Type I error is common in the literature (Creswell, 2008). However, incorrect conclusions might also be drawn if alpha level is too rigorous for individual tests (Grice \& Iwasaki, 2007). Too conservative adjustments of the significance level for individual tests result in tests with reduced statistical power; therefore, the likelihood that the tests will identify true differences is reduced (Schochet, 2008). 
Figure 2: Predictors of Perceived Overall Value of Higher Education, Personal Outcomes, and Professional Outcomes

\begin{tabular}{|c|c|c|}
\hline Category & $\begin{array}{l}\text { Items predictive of } \\
\text { outcomes }\end{array}$ & $\begin{array}{l}\text { Items not predictive of } \\
\text { outcomes }\end{array}$ \\
\hline $\begin{array}{l}\text { Goals for } \\
\text { coming to } \\
\text { the U.S. }\end{array}$ & $\begin{array}{l}\text { a) Learn to adjust to new } \\
\text { social and cultural } \\
\text { customs } \\
\text { a) Become more } \\
\text { independent } \\
\text { a) Get good quality } \\
\text { education }\end{array}$ & $\begin{array}{l}\text {-Learn new ways of thinking } \\
\text { and acting in field } \\
\text {-Get practical experience } \\
\text {-Develop skills to get a good job } \\
\text {-Get practice-oriented education } \\
\text {-Learn to work in a cross- } \\
\text { cultural environment } \\
\text {-Meet professionals in the field } \\
\text {-Improve English } \\
\text {-Learn about different cultural } \\
\text { viewpoints } \\
\text {-Build intercultural friendships } \\
\text {-Understand myself better } \\
\text {-Keep family happy }\end{array}$ \\
\hline $\begin{array}{l}\text { Institutional } \\
\text { support }\end{array}$ & $\begin{array}{l}\text { a) b) I am taught skills for } \\
\text { employment } \\
\text { a) c) Profs/staff encourage } \\
\text { contact among students } \\
\text { from different } \\
\text { backgrounds } \\
\text { a) It is easy to make } \\
\text { friends with intl students } \\
\text { b) I have opportunities to } \\
\text { apply my knowledge } \\
\text { c) Other students willing } \\
\text { to help intl students }\end{array}$ & $\begin{array}{l}\text {-Profs/ staff willing to help intl } \\
\text { students with academic } \\
\text { difficulties } \\
\text {-Easy to develop relationships } \\
\text { with profs and staff } \\
\text {-Profs/staff willing to help intl } \\
\text { students with cross-cultural } \\
\text { communication issues } \\
\text {-Easy to make friends with U.S. } \\
\text { students } \\
\text {-Profs/staff understand needs of } \\
\text { intl students } \\
\text {-I am encouraged to participate } \\
\text { in co-curricular activities }\end{array}$ \\
\hline $\begin{array}{l}\text { Engagement } \\
\text { in goal } \\
\text { achievement }\end{array}$ & $\begin{array}{l}\text { a) Talked about career } \\
\text { plans with } \\
\text { professor/advisor } \\
\text { a) c) Had serious } \\
\text { conversations with } \\
\text { students different from me } \\
\text { a) b) c) Used career } \\
\text { services } \\
\text { c) Used student services }\end{array}$ & $\begin{array}{l}\text {-Made friends with other intl } \\
\text { students } \\
\text {-Asked questions /contributed to } \\
\text { discussions } \\
\text {-Made friends with U.S. } \\
\text { students } \\
\text {-Relationships with people } \\
\text { outside university } \\
\text {-Asked profs/advisors for help }\end{array}$ \\
\hline
\end{tabular}


with academic or language

difficulties

-Involved in org made up of intl students

-Asked U.S. students for help

with academic or language

difficulties

-Asked other intl students for

help with academic or language

difficulties

-Involved in org made up of

U.S. students

a) Predictors of perceived overall value of U.S. higher education

b) Predictors of value related to professional outcomes

c) Predictors of value related to personal outcomes

This study was exploratory rather than confirmatory; relationships within data were examined to identify potential impacts and differences. Results from this study are preliminary and may be used to identify hypotheses tested more rigorously in future studies and replications (Schochet, 2008). In this context, the level of significance was not adjusted downward, in line with Schochet's (2008) argument that multiplicity adjustments are not required for exploratory analyses.

\section{DISCUSSION AND CONCLUSIONS}

Our study contributes to existing literature related to international students' experiences, particularly in regard to their goals, outcomes, professional and social engagement, and perception of institutional support. Findings regarding students' goals corroborate limited research indicating that international students are mostly motivated by academic and career outcomes (Hazen \& Alberts, 2006; Obst \& Forster, 2007). In our study, international students were quite engaged professionally, academically, and socially, which is consistent with previous research (Grayson, 2008). Yet, many students did not participate in student organizations made up mostly of domestic peers, which is contrary to an earlier study by Breuning (2007), who found that most international students were involved in student organizations made up of U.S. students. Finally, our participants were not actively engaged in using career services, which confirms previous research (Singaravelu, White, \& Bringaze, 2005).

Our participants believed that it was easy to make friends with other international peers, which corroborates other researchers' conclusions that 
the shared experience of being treated as a member of an outgroup creates a sense of identification with other international students, facilitates a sense of belonging, provides a supportive social and academic network, and alleviates stress related to negative experiences (Montgomery \& McDowell, 2009; Urban et al., 2010). Although our participants indicated that they often engaged in interactions with U.S. students, they expressed difficulties with making friends with them, which is in line with extant research (Hanassab, 2006; Spencer-Rogers, 2001; Urban et al., 2010).

Participants in our study thought that they received adequate academic support from professors and staff, and that they were learning skills needed for employment. However, they did not think that their institution was supporting them to engage in co-curricular activities. Moreover, respondents felt that their needs as international students were not well understood by professors and staff. This finding confirms previous studies suggesting that international students' problems and situations are often not accurately understood by administrators, staff, and faculty (Galloway \& Jenkins, 2005; Sherry et al., 2004).

In our study, professional outcomes were significantly lower than students' expectations related to professional development; whereas, students' personal outcomes were significantly higher than their pre-sojourn personal needs. Overall, the areas in which the students have gained the most included getting a good quality education, becoming more independent, and learning new ways of thinking and acting in the field of study. The least gains were in the areas of getting practical experience, understanding themselves better, and learning to write clearly and effectively. The achievement of students' professional outcomes was related to having opportunities to apply knowledge, being taught skills for employment, talking about career plans with professors or advisors, and using career services. Personal outcomes were related to having professors and staff encourage contact among students from different backgrounds, using career services, other students' willingness to help international students, having serious conversations with students very different from oneself, and using student services.

These findings enrich existing literature about international students' professional and personal expectations and outcomes by providing specific examples of the areas in which students benefit the most and the least as well as suggesting potential areas of students' experiences that can be impacted to increase their personal and professional gains. Furthermore, our study extends existing research suggesting that most international students are quite concerned about career-related issues such as career planning, getting work experience, and gaining job search skills ( Galloway \& Jenkins, 2005; Singaravelu et al., 2005). 
In our study, perceived value of U.S. higher education was related to students' goals, institutional support, and students' engagement in goal achievement. Specifically, among students' goals, there was a significant relationship among three reasons for coming to the United States (learning to adjust to new social and cultural customs, becoming more independent, and getting quality education) and students' perceived value of U.S. higher education. As far as institutional support, there was a significant relationship between three areas (being taught skills needed for future employment, having professors and staff encourage contact among students from different backgrounds, and having an environment where it is easy to make friends with other international students) and perceived value. In terms of students' engagement in goal achievement, there was a significant relationship between three areas (talking about career plans with professors or advisors, having serious conversations with students with differing backgrounds, and using career services) and perceived value of higher education.

\section{IMPLICATIONS}

Our research offers some areas of potential interventions that can help higher education leaders systematically address some contextual factors and positively impact how international students perceive their experience. These implications and recommendations are offered with the caveat that this study was limited to the convenience sample of international students at one public university, so the results cannot be generalized to the population of all international students.

\section{Addressing Difficulties with Obtaining Practical Experience}

Most international students desire to engage in professional activities such as on-campus work, internships, professional conferences, research, volunteering, and co-curricular activities; however, the status as an international student implies numerous barriers that limit access to such opportunities. Although contextual factors such as U.S. regulations regarding the employment of foreign nationals cannot be directly influenced by higher education institutions, advocating for this student population among employers and policy-makers can help educate them on the benefits of being systemically inclusive of international students and providing them with opportunities to gain practical experience. Universities can enhance international students' practical experiences by ensuring that they have equal access to participation in on-campus employment, research, and other hands-on projects. Part of this effort requires adequately preparing international students for such engagement in terms of teaching them to 
appropriately communicate their skills, their field of study, and potential unique contributions through resumes and interviewing, as well as preparing them to work in an environment governed by implicit and explicit cultural and social customs that might be very different from those to which students are accustomed.

In addition to facilitating opportunities for a practical application of academic knowledge, institutions can also impact students' professional success by ensuring that the skills taught during their course of study are relevant to employers, and by encouraging career-related conversations across campus. Offering professional development workshops for faculty, staff, and campus supervisors of student employees can help campus community better understand the needs, challenges, and contributions of international students and potential barriers to their academic progress, professional development, and social integration.

Since international students' professional outcomes are much lower than expected, it is crucial for higher education leaders to identify and address the environmental factors that hinder the achievement of students' professional goals. Part of such an effort should involve addressing the question whether those goals are realistic and whether opportunities for their achievement exist. For instance, in terms of students' goals, it might be worthwhile to explore how students' expectations might be influenced prior to their sojourn, so the inconsistencies between expectations and realities are minimized. One way to positively impact students' perceptions of their U.S. higher education experience is to provide opportunities to engage in interactions through social media with domestic and international students as well as staff at the target campus to clarify concerns about social and cultural customs, the reality of living independently in a foreign country for an extended period of time, the quality of the educational experiences in the U.S., and the realities concerning employment. Creating and sustaining a solid online network of international alumni could help prospective and current international students receive information related to the value of getting involved with student services and co-curricular activities as well as gaining practical experience.

\section{Addressing Challenges of Developing Meaningful Relationships with U.S. Students}

Higher education institutions can ensure that international students benefit personally from their experience by providing abundant opportunities for international students to create friendships and meaningfully interact with other international and domestic students as well as creating a supportive environment where international and domestic students use each other as academic resources and learn about their 
respective realities and viewpoints. Such efforts could involve facilitating early opportunities for interactions among international and domestic students during orientation programs instead of separating the students into two distinct programs. Living learning communities in residence halls can serve as an effective conduit to connecting international and domestic students who share academic interests. Peer programs that match international and domestic students could also contribute to positive experiences provided sufficient preparation and cross-cultural training is offered to both U.S. American and international students (Geelhoed, Abe, \& Talbot, 2003). In the classroom faculty have opportunities to create dynamics that provide a platform for international students to equally contribute to the dialogue and demonstrate their unique strengths while participating in group discussions (Cruickshank, Chen, \& Warren, 2012).

Facilitating co-curricular involvement is an area of opportunity for higher education institutions that want to help students accomplish several goals simultaneously. While involvement in culturally-based student organizations might positively affect student satisfaction and identification with the university, international students should also participate in student organizations related to their professional interests and made up equally or mostly of U.S. students. Such engagement can lead to much-needed interactions with domestic peers as students work in multicultural teams, apply their academic knowledge, and gain practical experience working on professional projects while simultaneously gaining - and contributing to $-\mathrm{a}$ greater cross-cultural learning. Although cross-cultural interactions occur mostly at the personal and informal level, the institutional level is the most crucial in creating the structures to facilitate increased interactions between international and domestic students.

The suggestions for higher education leaders, which emerged from our findings, have the potential to positively affect international students' perceptions of U.S. higher education and increase their professional and personal outcomes. Ultimately, meaningful engagement of international students can contribute to the recruitment and retention of international talent.

\section{REFERENCES}

Alberts, H. C. (2007). Beyond the headlines: Changing patterns in international student enrollment in the United States. GeoJournal, 68, 141-153.

Allen, H. W. (2010). Language-learning motivation during short-term study abroad: An activity theory perspective. Foreign Language Annals, 43, 27-49. 
Arthur, N. (2008). Counseling international students. In P. B. Pedersen, J. G. Draguns, W. J. Lonner, \& J. E. Trimble (Eds.), Counseling across cultures ( $6^{\text {th }}$ ed., pp. 275-289). Los Angeles, CA: Sage.

Bartram, B. (2007). The sociocultural needs of international students in higher education: A comparison of staff and student views. Journal of Studies in International Education, 11(2), 205-214.

Breuning, M. (2007). Undergraduate international students: A resource for the intercultural education of American peers? College Student Journal, 41(4), 1114-1122.

Chow, P. (2011). What international students think about U.S.

higher education: Attitudes and perceptions of prospective students in Africa, Asia, Europe and Latin America. New York, NY: Institute of International Education, Inc.

Constantine, M. G., Okazaki, S., \& Utsey, S. O. (2004). Self-concealment, social self-efficacy, acculturative stress, and depression in African, Asian, and Latino international college students. American Journal of Orthopsychiatry, 74, 230-241.

Creswell, J. W. (2008). Educational research: Planning, conducting, and evaluating quantitative and qualitative research ( ${ }^{\text {rd }}$ ed.). Upper Saddle River, NJ: Pearson.

Crockett, S. A., \& Hays, D. G. (2011). Understanding and responding to the career counseling needs of international college students on U.S. campuses. Journal of College Counseling, 14, 65-79.

Cruickshank, K., Chen, H., \& Warren, S. (2012). Increasing international and domestic student interaction through group work: A case study from the humanities. Higher Education Research and Development, 31(6), 797-810.

Dillman, D. A., Smyth, J. D., \& Christiam, L. M. (2009). Internet, mail and mixed-mode surveys: The tailored design method ( $3^{\text {rd }}$ ed.). Hoboken, NJ: Wiley.

Gall, M. D., Gall, J. P., \& Borg, W. R. (2007). Educational research: An introduction (8th ed.). Boston, MA: Pearson.

Galloway, F. J., \& Jenkins, J. R. (2005). The adjustment problems faced by international students in the United States: A comparison of international students and administrative perceptions at two private, religiously affiliated universities. NASPA Journal, 42(2), 175-187.

Geelhoed, R. J., Abe, J., \& Talbot, D. M. (2003). A qualitative investigation of U.S. students' experiences in an international peer program. Journal of College Student Development, 44(1), 5-17.

Grayson, J. P. (2008). The experiences and outcomes of domestic and 
international students at four Canadian universities. Higher Education Research and Development, 27(3), 215-230.

Grice, J. W., \& Iwasaki, M. (2007). A truly multivariate approach to MANOVA. Applied Multivariate Research, 12(3), 199-226.

Hanassab, S. (2006). Diversity, international students, and perceived discrimination: Implications for educators and counselors. Journal of Studies in International Education, 10(2), 157-172.

Hazen H. D., \& Alberts, H. C. (2006). Visitors or immigrants? International students in the United States. Population Space and Place, 12, 201216.

Ho, E., Bulman-Fleming, B., \& Mitchell, B. (2003). Course internationalization: Engaging students as learning resources. University of Waterloo, Ontario: Canadian Bureau for International Education, 2003 Annual Conference. Retrieved from: www.cte.uwaterloo.ca/grants/Internationalization/CourseInternation alization.pdf

Institute of International Education. (2013). Open doors 2013: International students in the United States. Washington DC: Institute of International Education. Retrieved from http://www.iie.org/Research-and-Publications/Open-Doors/Data Montgomery, C., \& McDowell, L. (2009). Social networks and the international student experience: An international community of practice? Journal of Studies in International Education, 13(4), 455466.

National Survey of Student Engagement (2005). The NSSE Annual Report. Bloomington, Indiana: Indiana University, Center for Postsecondary Research. Retrieved from

http://nsse.iub.edu/NSSE_2005_Annual_Report/surveyinstrument.cf $\mathrm{m}$ ?tab=Survey\%20Instrument

Obst, D., \& Forster, J. (2007). Perceptions of European higher education in third countries: Country report: USA. New York, NY: Institute of International Education.

Olivas, M., \& Li, C. (2006). Understanding stressors of international students in higher education: What college counselors and personnel need to know. Journal of Instructional Psychology, 33(3), 217-222.

Reynolds, A. L., \& Constantine, M. G. (2007). Cultural adjustment difficulties and career development of international college students. Journal of Career Assessment, 15(3), 338-350.

Rosser, V., Hermsen, J. M., Mamiseishvili, K., \& Wood, M. S. (2007). A national study examining the impact of SEVIS on international student and scholar advisors. Higher Education, 54, 525-542.

Schochet, P. Z. (2008). Technical methods report: Guidelines for multiple 
testing in impact evaluations (NCEE 2008-4018). Washington, DC: National Center for Education Evaluation and Regional Assistance, Institute of education Sciences, U.S. Department of Education.

Sherry, C., Bhat, R., Beaver, B., \& Ling, A. (2004). Students as customers:

The expectations and perceptions of local and international students. Paper presented at the HERDSA 2004 Conference, Sarawak.

Singaravelu, H. D., White, L. J., \& Bringaze, T. B. (2005). Factors influencing international students' career choice. Journal of Career Development, 32(1), 46-59.

Spencer-Rogers, J. (2001). Consensual and individual stereotypic beliefs about international students among American host nationals. International Journal of Intercultural Relations, 25, 639-657.

Trice, A. G. (2004). Mixing it up: International graduate students' social interactions with American students. Journal of College Student Development, 45(6), 671-687.

Urban, E. (2012). Value of U.S. higher education for international students in the context of higher education internationalization (Doctoral Dissertation). Retrieved from http://scholarworks.wmich.edu/dissertations/124.

Urban, E., Orbe, M. P., Tavares, N. A., \& Alvarez, W. (2010). Exploration of Dominican international students' experiences. Journal of Student Affairs Research and Practice, 47(2), 233-250.

Yeh, J. C., \& Inose, M. (2003). International students’ reported English fluency, social support satisfaction, and social connectedness as predictors of acculturative stress. Counseling Psychology Quarterly, 16(1), 15-28.

EWA URBAN, Ph.D. is an associate director for assessment in Career and Student Employment Services at Western Michigan University. Her research interests include intercultural communication and international students' higher education experiences, learning and career outcomes, and career transitions. Email: ewa.l.urban@wmich.edu

LOUANN BIERLEIN PALMER, Ed.D., is a professor of educational leadership and the Ph.D. program coordinator for the Department of Educational Leadership, Research and Technology at Western Michigan University. Her research interests cover a wide span of education and social policy issues. Email: LBierleinPalmer@wmich.edu 